\title{
Sistematização da Assistência de Enfermagem nas Unidades de Saúde: Uma revisão integrativa
}

\author{
Systematization of Nursing Care in Health Units: An integrative review \\ Sistematización de la Atención de Enfermería en Unidades de Salud: Una revisión integradora
}

Recebido: 11/03/2021 | Revisado: 18/03/2021 | Aceito: 23/03/2021 | Publicado: 01/04/2021

Gabriela Martins

ORCID: https://orcid.org/0000-0003-4118-2536 Universidade do Vale do Taquari, Brasil E-mail: gabriela.martins@universo.univates.br

Arlete Eli Kunz da Costa ORCID: https://orcid.org/0000-0002-5655-3646 Universidade do Vale do Taquari, Brasil E-mail: arlete.costa@univates.br

Fernanda dos Santos

ORCID: https://orcid.org/0000-0001-9153-665X Universidade Federal de Pelotas, Brasil Email:fernanda.santos5@univates.br

\begin{abstract}
Resumo
Objetivo: Analisar artigos brasileiros sobre a Sistematização da Assistência de Enfermagem (SAE). Metodologia: revisão integrativa, com abordagem narrativa. A coleta de dados foi por meio de buscas nas bases de dados Scielo (Scientific Electronic Library Online) e Lilacs (Literatura Latino-americana e do Caribe em Ciências da Saúde) utilizando-se os termos "Enfermagem", "Processo de Enfermagem"," "Centros de Saúde", "Equipe de Enfermagem", “Cuidados de Enfermagem", "ILP” e "Atenção Básica”, artigos publicados até 2021. Resultados: Foram elencados e analisados dezesseis artigos, que contemplaram a questão norteadora e os critérios de inclusão. O estudo serviu para salientar a importância da SAE nas Unidades de Saúde, bem como, destaca quais foram os meios de implementação utilizados nos artigos pesquisados. Conclusão: Este estudo evidenciou nos artigos publicados entre 2004 e 2021, sendo que em todos foram apontadas dificuldades e possíveis soluções para a aplicação da SAE nas Unidades de Saúde. Apesar dos obstáculos que os(as) enfermeiros(as) enfrentam para implementar a SAE, suas contribuições significativas devem ser levadas em conta, propondo um momento de reflexão pelos profissionais de enfermagem, especialmente os(as) enfermeiros(as). A Sistematização da Assistência de Enfermagem é compromisso e humanização do cuidado, sendo de grande valia o esforço para implementá-la nas unidades básicas, ILPS e unidades hospitalares.

Palavras-chave: Enfermagem; Processo de enfermagem; Centros de saúde; Equipe de enfermagem; Cuidados de enfermagem; ILP; Atenção básica.
\end{abstract}

\begin{abstract}
Objective: To analyze Brazilian articles on the Systematization of Nursing Assistance (SAE). Methodology: integrative review, with a narrative approach. Data collection was performed by searching the Scielo (Scientific Electronic Library Online) and Lilacs (Latin American and Caribbean Literature in Health Sciences) databases using the terms "Nursing", "Nursing Process", "“ Health Centers ”, Nursing Team ”,“ Nursing Care ”,“ ILP "and" Primary Care ”, articles published until 2021. Results: Sixteen articles were listed and analyzed, which addressed the guiding question and criteria of inclusion. The study served to highlight the importance of SAE in Health Units, as well as highlighting the means of implementation used in the researched articles. Conclusion: This study showed in the articles published between 2004 and 2021, and in all of them difficulties and possible solutions for the application of SAE in Health Units were pointed out. Despite the obstacles that nurses face to implement SAE, their significant contributions must be taken into account, proposing a moment for reflection by nursing professionals, especially nurses. The Systematization of Nursing Assistance is a commitment and humanization of care, and the effort to implement it in basic units, ILPS and hospital units is of great value.
\end{abstract}

Keywords: Nursing; Nursing process; Health centers; Nursing team; Nursing care; ILP; Primary care.

\section{Resumen}

Objetivo: Analizar artículos brasileños sobre Sistematización de la Asistencia de Enfermería (SAE). Metodología: revisión integradora, con enfoque narrativo. La recolección de datos se realizó mediante la búsqueda en las bases de datos Scielo (Scientific Electronic Library Online) y Lilacs (Literatura Latinoamericana y del Caribe en Ciencias de la Salud) utilizando los términos "Enfermería", "Proceso de Enfermería", "Centros de Salud", "Equipo de Enfermería", "Atención de enfermería", "PIA" y "Atención primaria", artículos publicados hasta 2021. Resultados: Se enumeraron 
y analizaron 16 artículos, que abordaron la pregunta orientadora y los criterios de inclusión. El estudio sirvió para resaltar la importancia del SAE en las Unidades de Salud, así como destacar los medios de implementación utilizados en los artículos investigados. Conclusión: Este estudio se mostró en los artículos publicados entre 2004 y 2021, y en todas las dificultades y posibles soluciones para la aplicación del SAE en las Unidades de Salud, a pesar de los obstáculos que enfrentan las enfermeras para implementar el SAE, sus importantes aportes deben ser asumidos. en cuenta, proponiendo un momento de reflexión por parte de los profesionales de enfermería, especialmente enfermeras. La Sistematización de la Atención de Enfermería es un compromiso y humanización del cuidado, y el esfuerzo para implementarlo en unidades básicas, ILPS y unidades hospitalarias es de gran valor.

Palabras clave: Enfermería; Proceso de enfermería; Centros de salud; Equipo de enfermería; Atención de enfermería; ILP; Atención primaria.

\section{Introdução}

A Sistematização da Assistência de Enfermagem é regulamentada no Brasil pela Resolução nº 358/2009 do Conselho Federal de Enfermagem (Cofen, 2009), preconizando que sua implantação deve ocorrer em todas as unidades de atendimento à saúde que ofereçam assistência de enfermagem (Barreto, 2020). A Resolução do Conselho Federal de Enfermagem estabelece que o processo de enfermagem deve ser realizado de modo deliberado e sistemático em todos os ambientes públicos ou privados em que ocorre o cuidado profissional de enfermagem. Ainda destaca as cinco etapas: coleta de dados (ou histórico), diagnóstico, planejamento, implementação e avaliação (Cofen, 2009).

A utilização desse instrumento garante ao enfermeiro identificar as necessidades de cada paciente/grupo, direcionando o atendimento a partir de prioridades estabelecidas, o que favorece a implementação de cuidados holísticos, integrais e personalizados, segundo Chaves et al., (2016). Apesar da operacionalização da SAE ser obrigatória nas instituições brasileiras que abrigam os serviços de enfermagem, sua aplicação, muitas vezes, não é empreendida de forma correta ou completa (Barreto, 2020).

De acordo com Oliveira (2019) uma das problemáticas em torno da SAE é que, apesar de ela ter regulamentos e publicações que a amparam, ainda é comum o relato, por parte dos enfermeiros, sobre dificuldades na aplicação da SAE na prática diária do cuidar, bem como de percebê-la como um meio para otimizar o cuidado clínico de enfermagem. Muitos acadêmicos, e até mesmo profissionais, com vários anos de experiência, ainda confundem e/ou sumarizam a SAE como mero instrumento de coleta de dados, não compreendendo que esta vai muito além de uma atividade burocrática. Outros ainda a associam a simples formulários e a mais uma atividade de registro, entre tantas realizadas pelos enfermeiros.

Paralelamente, para a Enfermagem, o descaso com o registro do processo de cuidado, seja no prontuário do paciente, ou em outros documentos próprios, pode resultar, por um lado, em ausência de visibilidade e de reconhecimento profissional e, por outro lado, em obstáculo para a avaliação de sua prática, o que é talvez mais sério, pois dificulta o avanço da ciência de Enfermagem (Garcia, 2016).

Portanto, identificar os desafios enfrentados pelos enfermeiros para implementar a SAE em sua rotina de trabalho e apontar a forma como estes têm conseguido, ao menos em parte, operacionalizar a SAE, acredita-se que os resultados possam ser úteis para favorecer a discussão da temática e frisar a relevância da aplicação da SAE (Barreto, 2020).

\section{Metodologia}

Trata-se de uma pesquisa de revisão integrativa com artigos publicados até 2021, buscados nas bases de dados Scielo (Scientific Electronic Library Online) e Lilacs (Literatura Latino-americana e do Caribe em Ciências da Saúde), abordando o tema da Sistematização da Assistência de Enfermagem nas Unidades de Saúde, utilizando-se os termos "Enfermagem", "Processo de Enfermagem", “Centros de Saúde", "Equipe de Enfermagem”, “Cuidados de Enfermagem”, "ILP” e "Atenção Básica”. Os resultados esperados desta pesquisa servirão para salientar a importância da SAE nas Unidades de Saúde, bem como, destacar quais foram os meios de implementação utilizados nos artigos pesquisados. 


\section{Resultados e Discussão}

Na presente revisão integrativa da literatura, foram analisados dezesseis artigos, que contemplaram a questão norteadora e os critérios de inclusão, sendo que eles foram analisados na íntegra, com o propósito de serem caracterizados, interpretados e discutidos. Os artigos selecionados estão inicialmente apresentados e brevemente caracterizados no quadro sinóptico abaixo (Matiello, Costa, Lohmann, Lavall, 2020).

Conforme o Quadro Sinóptico 1.

Quadro 1. Artigos selecionados.

\begin{tabular}{|c|c|c|c|c|c|}
\hline $\mathbf{N}^{\circ}$ & $\begin{array}{c}\text { Base de } \\
\text { Dados }\end{array}$ & Autor(s)/Ano & Título & Periódico & Objetivos do estudo \\
\hline I & Scielo & $\begin{array}{l}\text { Santana, } \\
\text { Edileuza Teixeira } \\
\text { et al. (2021) }\end{array}$ & $\begin{array}{l}\text { Diagnósticos de enfermagem } \\
\text { da taxonomia NANDA-I para } \\
\text { idosos em instituição de longa } \\
\text { permanência. }\end{array}$ & $\begin{array}{l}\text { Esc. Anna } \\
\text { Nery }\end{array}$ & $\begin{array}{l}\text { Identificar diagnósticos de } \\
\text { enfermagem da Taxonomia da } \\
\text { NANDA-I em idosos } \\
\text { institucionalizados. }\end{array}$ \\
\hline II & Scielo & $\begin{array}{l}\text { Gutiérrez, Maria } \\
\text { GabyRivero de; } \\
\text { Morais, Sheila } \\
\text { Coelho Ramalho } \\
\text { Vasconcelos. } \\
\text { (2017) }\end{array}$ & $\begin{array}{l}\text { Sistematização da Assistência } \\
\text { de Enfermagem e a formação } \\
\text { da identidade profissional. }\end{array}$ & $\begin{array}{l}\text { Rev. Bras. } \\
\text { Enferm. }\end{array}$ & $\begin{array}{l}\text { Explorar os argumentos que ampliem a } \\
\text { compreensão dos possíveis nexos entre } \\
\text { a sistematização da assistência de } \\
\text { enfermagem e a formação da } \\
\text { identidade profissional. Para tanto, } \\
\text { abordam-se alguns aspectos } \\
\text { relacionados a esses temas, destacando } \\
\text { questões referentes às diferenças na } \\
\text { concepção da sistematização da } \\
\text { assistência de enfermagem e do } \\
\text { processo de enfermagem, bem como } \\
\text { na prática dessa atividade e sua } \\
\text { possível repercussão no } \\
\text { estabelecimento de sua relação com a } \\
\text { identidade profissional. }\end{array}$ \\
\hline III & Scielo & $\begin{array}{l}\text { Oliveira, Marcos } \\
\text { Renato de et } \\
\text { al. (2019) }\end{array}$ & $\begin{array}{l}\text { Sistematização da assistência } \\
\text { de enfermagem: percepção e } \\
\text { conhecimento da enfermagem } \\
\text { Brasileira. }\end{array}$ & $\begin{array}{l}\text { Rev. Bras. } \\
\text { Enferm. }\end{array}$ & $\begin{array}{l}\text { Apresentar a percepção e o } \\
\text { conhecimento de enfermeiros e } \\
\text { acadêmicos de enfermagem brasileiros } \\
\text { quanto à Sistematização da Assistência } \\
\text { de Enfermagem. }\end{array}$ \\
\hline IV & Scielo & $\begin{array}{l}\text { Salvador, Ptala } \\
\text { Tuani Candido } \\
\text { de Oliveira et al. } \\
(2018)\end{array}$ & $\begin{array}{l}\text { Validação de objeto virtual de } \\
\text { aprendizagem para apoio ao } \\
\text { ensino da sistematização da } \\
\text { assistência de enfermagem. }\end{array}$ & $\begin{array}{l}\text { Rev. Bras. } \\
\text { Enferm }\end{array}$ & $\begin{array}{l}\text { Descrever o processo de validação de } \\
\text { conteúdo de um Objeto Virtual de } \\
\text { Aprendizagem para apoio ao ensino da } \\
\text { sistematização da assistência de } \\
\text { enfermagem aos técnicos em } \\
\text { enfermagem. }\end{array}$ \\
\hline
\end{tabular}




\begin{tabular}{|c|c|c|c|c|c|}
\hline V & Scielo & $\begin{array}{l}\text { Barreto, Mayckel } \\
\text { da Silva } \text { et al. } \\
(2020)\end{array}$ & $\begin{array}{l}\text { Sistematização da assistência } \\
\text { de enfermagem: a práxis do } \\
\text { enfermeiro de hospital de } \\
\text { pequeno porte. }\end{array}$ & $\begin{array}{l}\text { Esc. Anna } \\
\text { Nery }\end{array}$ & $\begin{array}{l}\text { Descrever a vivência dos enfermeiros } \\
\text { atuantes em unidade hospitalar em } \\
\text { relação à Sistematização da } \\
\text { Assistência de Enfermagem. }\end{array}$ \\
\hline VI & Scielo & $\begin{array}{l}\text { Jesus, Isac Silva } \\
\text { de et al. (2010) }\end{array}$ & $\begin{array}{l}\text { Cuidado sistematizado a idosos } \\
\text { com afeccão demencial } \\
\text { residentes em instituição de } \\
\text { longa permanência. }\end{array}$ & $\begin{array}{l}\text { Rev. Gaúcha } \\
\text { Enferm. }\end{array}$ & $\begin{array}{l}\text { O estudo objetivou sistematizar um } \\
\text { modelo básico de cuidados de } \\
\text { enfermagem baseado na taxonomia da } \\
\text { North American Nursing Diagnosis } \\
\text { Association (NANDA), testado em } \\
\text { idosos com afecção demencial, } \\
\text { residentes em Instituição de Longa } \\
\text { Permanência (ILPI), no interior } \\
\text { baiano. }\end{array}$ \\
\hline VII & Lilacs & \begin{tabular}{|l} 
Somariva, Vaness \\
a Cristina; \\
Birolo, Ioná \\
Vieira; Tomasi, \\
Cristiane \\
Damiani; \\
Soratto, Jacks. \\
$(2019)$
\end{tabular} & $\begin{array}{l}\text { Percepções das equipes de } \\
\text { enfermagem na Atenção } \\
\text { Básica frente a Sistematização } \\
\text { da Assistência de } \\
\text { Enfermagem. }\end{array}$ & Enferm. Foco & $\begin{array}{l}\text { Avaliar a Sistematização da } \\
\text { Assistência de Enfermagem (SAE) na } \\
\text { Atenção Básica de um município do } \\
\text { extremo sul catarinense, frente às } \\
\text { percepções das equipes de } \\
\text { enfermagem. }\end{array}$ \\
\hline VIII & Scielo & $\begin{array}{l}\text { Ribeiro, Grasielle } \\
\text { Camisão; } \\
\text { Padoveze, Maria } \\
\text { Clara. (2018) }\end{array}$ & $\begin{array}{l}\text { Sistematização da Assistência } \\
\text { de Enfermagem em unidade } \\
\text { básica de saúde: percepção da } \\
\text { equipe de enfermagem. }\end{array}$ & $\begin{array}{l}\text { Rev. esc. } \\
\text { enferm. USP }\end{array}$ & $\begin{array}{l}\text { Realizar um diagnóstico situacional da } \\
\text { Sistematização da Assistência de } \\
\text { Enfermagem (SAE) em uma Unidade } \\
\text { Básica de Saúde (UBS), na percepção } \\
\text { da equipe de enfermagem. }\end{array}$ \\
\hline IV & Scielo & $\begin{array}{l}\text { Silva, Elisama } \\
\text { Gomes Correia et } \\
\text { al (2011) }\end{array}$ & $\begin{array}{l}\text { O conhecimento do enfermeiro } \\
\text { sobre a Sistematização da } \\
\text { Assistência de Enfermagem: } \\
\text { da teoria à prática. }\end{array}$ & $\begin{array}{l}\text { Rev. esc. } \\
\text { enferm. }\end{array}$ & $\begin{array}{l}\text { Analisar o conhecimento dos } \\
\text { enfermeiros sobre a Sistematização da } \\
\text { Assistência de Enfermagem (SAE) em } \\
\text { um hospital de grande porte em Recife, } \\
\text { Pernambuco. }\end{array}$ \\
\hline$X$ & Scielo & $\begin{array}{l}\text { Hermida, Patrícia } \\
\text { Madalena Vieira; } \\
\text { Araujo, Izilda } \\
\text { Esmênia } \\
\text { Muglia. (2006) }\end{array}$ & $\begin{array}{l}\text { Sistematização da assistência } \\
\text { de enfermagem: subsídios para } \\
\text { implantação. }\end{array}$ & $\begin{array}{l}\text { Rev. bras. } \\
\text { enferm., }\end{array}$ & $\begin{array}{l}\text { Identificar e refletir as fases do } \\
\text { planejamento para implantação dessa } \\
\text { prática, a fim de subsidiar a atuação do } \\
\text { enfermeiro nesse processo. }\end{array}$ \\
\hline XI & Scielo & $\begin{array}{l}\text { Bittar, Daniela } \\
\text { Borges; Pereira, } \\
\text { Lílian Varanda; } \\
\text { Lemos, Rejane } \\
\text { Cussi Assunção. } \\
(2006)\end{array}$ & $\begin{array}{l}\text { Sistematização da assistência } \\
\text { de enfermagem ao paciente } \\
\text { crítico: proposta de } \\
\text { instrumento de coleta de } \\
\text { dados. }\end{array}$ & $\begin{array}{l}\text { Texto contexto } \\
\text { - enferm. }\end{array}$ & $\begin{array}{l}\text { Elaborar um instrumento de coleta de } \\
\text { dados visando o registro de forma } \\
\text { eficiente e validá-lo em sua forma } \\
\text { aparente e de conteúdo. }\end{array}$ \\
\hline
\end{tabular}




\begin{tabular}{|c|c|c|c|c|c|}
\hline XII & Scielo & $\begin{array}{l}\text { Menezes, Silvia } \\
\text { Regina Tamae; } \\
\text { Priel, Margareth } \\
\text { Rose; Pereira, } \\
\text { Luciane Lucio. } \\
(2011)\end{array}$ & $\begin{array}{l}\text { Autonomia e vulnerabilidade } \\
\text { do enfermeiro na prática da } \\
\text { Sistematização da Assistência } \\
\text { de Enfermagem. }\end{array}$ & $\begin{array}{l}\text { Rev. esc. } \\
\text { enferm. USP }\end{array}$ & $\begin{array}{l}\text { Reconhecer a autonomia e a } \\
\text { vulnerabilidade do enfermeiro no } \\
\text { processo de implantação e } \\
\text { implementação da Sistematização da } \\
\text { Assistência de Enfermagem }\end{array}$ \\
\hline XIII & Scielo & $\begin{array}{l}\text { Amante, Lúcia } \\
\text { Nazareth; } \\
\text { Rosseto, } \\
\text { Annelise Paula; } \\
\text { Schneider, } \\
\text { Dulcinéia } \\
\text { Ghizoni. (2009) }\end{array}$ & $\begin{array}{l}\text { Sistematização da Assistência } \\
\text { de Enfermagem em Unidade } \\
\text { de Terapia Intensiva sustentada } \\
\text { pela Teoria de Wanda Horta. }\end{array}$ & $\begin{array}{l}\text { Rev. esc. } \\
\text { enferm. USP }\end{array}$ & $\begin{array}{l}\text { implementar a Sistematização da } \\
\text { Assistência de Enfermagem (SAE), } \\
\text { tendo como referencial a Teoria das } \\
\text { Necessidades Humanas Básicas de } \\
\text { Wanda de Aguiar Horta e o } \\
\text { Diagnóstico de Enfermagem da North } \\
\text { American Nursing Diagnosis } \\
\text { Association (NANDA) }\end{array}$ \\
\hline XIV & Scielo & $\begin{array}{l}\text { Soares, Mirelle } \\
\text { Inácio et AL } \\
(2015)\end{array}$ & $\begin{array}{l}\text { Sistematização da assistência } \\
\text { de enfermagem: facilidades e } \\
\text { desafios do enfermeiro na } \\
\text { gerência da assistência }\end{array}$ & $\begin{array}{l}\text { Esc. Anna } \\
\text { Nery }\end{array}$ & $\begin{array}{l}\text { Analisar as facilidades e os desafios do } \\
\text { enfermeiro na gerência da assistência } \\
\text { instrumentalizada pela Sistematização } \\
\text { da Assistência de Enfermagem (SAE). }\end{array}$ \\
\hline XV & Scielo & $\begin{array}{l}\text { Hermida, Patrícia } \\
\text { Madalena Vieira. } \\
(2004)\end{array}$ & $\begin{array}{l}\text { Desvelando a implementação } \\
\text { da Sistematização da } \\
\text { Assistência de Enfermagem. }\end{array}$ & $\begin{array}{l}\text { Rev. bras. } \\
\text { enferm. }\end{array}$ & $\begin{array}{l}\text { identificar as dificuldades encontradas } \\
\text { na implementação da SAE e os fatores } \\
\text { que interferem prejudicando sua } \\
\text { implementação, nos trabalhos } \\
\text { nacionais publicados nos últimos cinco } \\
\text { anos. }\end{array}$ \\
\hline XVI & Scielo & $\begin{array}{l}\text { Medeiros, Ana } \\
\text { Lúcia de; Santos, } \\
\text { Sérgio Ribeiro } \\
\text { dos; Cabral, } \\
\text { Rômulo } \\
\text { Wanderley de } \\
\text { Lima. (2012) }\end{array}$ & $\begin{array}{l}\text { Sistematização da assistência } \\
\text { de enfermagem na perspectiva } \\
\text { dos enfermeiros: uma } \\
\text { abordagem metodológica na } \\
\text { teoria fundamentada. }\end{array}$ & $\begin{array}{l}\text { Rev. Gaúcha } \\
\text { Enferm. }\end{array}$ & $\begin{array}{l}\text { Compreender, a partir da perspectiva } \\
\text { dos enfermeiros, a experiência de } \\
\text { vivenciar a Sistematização da } \\
\text { Assistência de Enfermagem (SAE) em } \\
\text { um serviço de obstetrícia. }\end{array}$ \\
\hline
\end{tabular}

Fonte: Autoras (2021).

Entre os dezesseis artigos selecionados e analisados, foram encontradas cinco publicações na Revista Brasileira de Enfermagem, três artigos publicados na Revista Escola Anna Nery, três publicações na Revista Escola de Enfermagem da USP, duas publicações na Revista Gaúcha de Enfermagem, uma publicação na Revista Enfermagem em Foco, um estudo publicado na Revista Texto e Contexto, e uma publicação na Revista Escola de Enfermagem.

Dos artigos selecionados, quinze são da base de dados Scielo, e um da base de dados Lilacs. Referente ao ano de publicação, o artigo I foi publicado em 2021, II em 2017, III e o VII em 2019, IV e o VIII em 2018, V em 2020, VI em 2010, IV e XII em 2011, X e XI em 2006, XIII em 2009, XIV, em 2015, XV em 2004 e XVI em 2012. 
Em relação ao delineamento de pesquisa, seis quantitativos, cinco estudos qualitativos e cinco revisões de literatura. Dos dezesseis artigos encontrados, três artigos abordam a SAE nas instituições hospitalares em diferentes setores como: Unidade de Terapia Intensiva, Internação e Maternidade. Dois artigos abordam a SAE nas Instituições de Longa Permanência e dois em atenção básica.

O artigo I, possui publicação recente e atualizada sobre os Diagnósticos de Enfermagem, segunda etapa do Processo de Enfermagem. Esse estudo mostra a importância do enfermeiro, sendo um profissional essencial nas ILPIs, já que por meio da aplicação do PE com vistas à implementação da SAE possibilita uma assistência sistematizada, individualizada, e holística impactando na preservação e/ou aumento da funcionalidade dos idosos institucionalizados, melhorando a sua qualidade de vida no processo de envelhecer (Santana, 2021). Ao reconhecer os idosos institucionalizados como um grupo vulnerável e específico, é ressaltada a relevância da Enfermagem na formulação do julgamento clínico e crítico direcionados à prevenção de danos, promoção da saúde e controle das possíveis complicações (Souza, Santana, Jesus, 2017).

O artigo II, Sistematização da Assistência de Enfermagem e a formação da identidade profissional, relaciona a identidade profissional com a aplicação da Sistematização da Assistência de Enfermagem. Ao lermos os diversos artigos sobre as dificuldades encontradas no ensino e na prática da SAE, que a nosso ver se referem ao PE, podemos verificar que grande parte deles diz respeito aos aspectos operacionais envolvidos na sua execução, tais como: quantidade de tempo necessária para fazer o processo de enfermagem, falta de tempo, instrumentos longos demais, consumo excessivo de tempo para preenchimento de toda a documentação exigida, entre outros. Alguns também referem problemas relativos às limitações dos profissionais no que diz respeito ao desenvolvimento de competências necessárias para a implementação de cada uma das etapas do PE (déficit de conhecimento de semiologia e das técnicas básicas para o exame físico, para o desenvolvimento de raciocínio clínico e terapêutico precisos, para o emprego da tecnologia de informação e comunicação, entre outros) (Carvalho et al, 2009).

Por isso, a existência de normas e regras profissionais que orientam o trabalho profissional é um dos atributos do status de profissão, a SAE está incluída nesse contexto. A SAE ou do PE e a identidade profissional não é simples de ser analisada, uma vez que se trata de uma prática profissional que apresenta uma série de ambiguidades e tensões, configurando um saber/fazer constituído não só de dificuldades, mas também e, principalmente, de potencialidades para consolidar-se como gerador e organizador de nossas práticas de cuidado, referencial de nossa profissão (Gutiérrez, Morais, 2017).

No artigo III, Sistematização da assistência de enfermagem: percepção e conhecimento da enfermagem Brasileira, percebe-se que a SAE e o PE são importantes pela maioria dos profissionais de enfermagem do Brasil, porém a aplicação efetiva de ambos na prática dos cuidados clínicos de enfermagem ainda é uma lacuna a ser superada. O estudo também aponta na direção de que o conhecimento do raciocínio clínico é essencial para uma atitude positiva frente à SAE (Oliveira et al, 2019). Já se sabe que, dentre as variáveis presentes para a não adoção de um processo sistematizado de cuidar encontram-se falhas de comunicação entre os profissionais (Gengo, et al, 2018), inadequado nível de conhecimento dos profissionais e quantidade inadequada de recursos humanos.

Por outro lado, o uso de tecnologias e empoderamento dos profissionais de enfermagem durante a implementação da SAE, são fatores que contribuem para o seu uso pelos profissionais (Kang, Hwang, Choi, 2019). De tal forma que alguns autores percebem o PE como uma tecnologia do cuidado, que orienta a sequência do raciocínio clínico e melhora a qualidade do cuidado (Dal Sasso, 2013).

O artigo IV, Validação de objeto virtual de aprendizagem para apoio ao ensino da sistematização da assistência de enfermagem, evidencia-se que a incorporação de tecnologias educacionais nos ambientes de ensino envolve dois aspectos primordiais: por um lado, um processo de construção e validação de material educativo com aporte técnico, pedagógico e metodológico adequados, e, por outro, a incorporação da tecnologia educacional validada com um necessário preparo docente e da instituição de ensino como um todo (Salvador et al, 2018). Assim, destaca-se que a validação de uma tecnologia educacional 
deve ser um processo frequente e contínuo, isso devido às constantes inovações e melhorias tecnológicas vivenciadas (Hohlfeld, Ritzhaupt, Barron, 2013).

$\mathrm{O}$ artigo $\mathrm{V}$, Sistematização da assistência de enfermagem: a práxis do enfermeiro de hospital de pequeno porte, nesse estudo, os participantes demonstraram desmotivação para o emprego da SAE, sobretudo, devido à falta de valorização e reconhecimento de tais ações pelos demais membros da equipe (Barreto, 2020). Foi possível perceber certa dificuldade em se estabelecer um eficiente processo de comunicação e um satisfatório relacionamento profissional entre o enfermeiro líder e a equipe de enfermagem, assim como identificou-se diferentes fatores dificultaram a implementação da SAE nas unidades hospitalares de pequeno porte, cenários deste estudo, tais como, sobrecarga de trabalho do enfermeiro, falta de documentos adequados para efetivar os registros, desinteresse da equipe para implementação e falta de apoio da instituição hospitalar e seus gestores. Isto faz com que a operacionalização da SAE ocorresse de forma fragmentada e parcial, principalmente por aqueles enfermeiros que reconheciam a relevância desta metodologia de trabalho (Barreto, 2020).

Desse modo, a sobrecarga que acomete os enfermeiros, especialmente àqueles inseridos em pequenos hospitais e que acabam por realizar também outras atividades/funções, não inclusas no rol de suas áreas de atuação, tem representado um obstáculo à qualificação do atendimento de enfermagem por meio da utilização da SAE (Medeiros, Santos, Cabral, 2013). Por conseguinte, o enfermeiro passa a não dispor de tempo para aplicar todas as etapas que envolvem a SAE de forma contínua, sistemática e adequada. Porém, a falta de tempo não é vista como um motivo sólido para impedir a implementação da SAE quando ela é colocada como prioridade (Domingos, 2017).

O artigo VI, Cuidado sistematizado a idosos com afecção demencial residentes em instituição de longa permanência, descreve a sistematização da assistência de enfermagem para idosos com afecção demencial ou com diagnóstico de demência já confirmado, residentes em uma ILPI de Jequié, BA. Inclui o processo de enfermagem na determinação do diagnóstico de enfermagem, com base nos preceitos da North American Nursing Diagnosis Association, os objetivos e as ações de enfermagem enquanto plano de assistência individualizada (Jesus, 2010).

A adoção da SAE nas instituições de longa permanência para idosos pode constituir-se na busca não apenas do atendimento do idoso institucionalizado, mas também a prestação de cuidados através de ações direcionadas aos seus familiares estabelecidos (Jesus, 2010). Desse modo, considerando os fatores que caracterizam e aqueles que se relacionam às condições de assistência e trabalho nas ILPIs de modo geral, foi possível testar e sistematizar um modelo básico de cuidados de enfermagem baseado em Nanda, aplicável aos idosos com afecção demencial ou com diagnóstico médico de demência estabelecido (Jesus, 2010).

Nesse contexto, os profissionais da enfermagem devem estar preparados para atender esta demanda populacional, uma vez que seu papel junto aos idosos especialmente após acidentes por quedas não se restringe à prestação de assistência às complicações decorrentes da queda, abrangendo também a oferta de apoio emocional a esse idoso e sua família (Pereira, Dos Santos, Rodrigues et al, 2016).

O artigo VII, Percepções das equipes de enfermagem na Atenção Básica frente a Sistematização da Assistência de Enfermagem, mostra claramente que a equipe de enfermagem de acordo com suas percepções frente à Sistematização da Assistência de Enfermagem, reconhecem de modo significativo os benefícios e os fatores facilitadores para sua implementação. É preciso enfatizar que quando realizado comparações entre os enfermeiros e técnicos de enfermagem, estes últimos apresentaram menor familiaridade de conhecimento acerca do que são e a qual a aplicabilidade da SAE e PE (Somariva, Birolo, Mariva, Tomasi, Soratto, 2019).

O desenvolvimento da SAE e PE é fragmentado, restrito a apenas algumas etapas operacionais, em geral na coleta de dados ou histórico de enfermagem, seguido da evolução de enfermagem. Isso prejudica a tomada de decisão da equipe, por não 
se sustentar em um planejamento sólido, baseado em evidências científicas, denotando uma certa resistência dos profissionais de enfermagem em aderir métodos reconhecidos legalmente (Somariva, Birolo, Mariva, Tomasi, Soratto, 2019).

Ao longo da discussão percebe-se que a SAE ainda não é vivenciada eficazmente na prática e, seria inviável sua plena inserção devido a falta de conhecimento dos profissionais, em especial da atenção básica, cuja visão da SAE ainda é limitada por não serem estimulados a trabalharem com esta prática (Amante, 2009).

Portanto, o estudo da SAE na teoria, bem como sua aplicação na prática evidencia a importância do profissional enfermeiro na assistência ao paciente. Destacando a relevância da assistência da enfermagem na atenção básica pois é neste local em que será possível conversar com o usuário de forma mais aberta, uma vez que é o ambiente mais frequentado por ele, quando comparado com a atenção especializada e a equipe da unidade conhece a sua população (Morais, Brasil, Tolfo, Costa, Lohmann, 2020).

O artigo VIII, Sistematização da Assistência de Enfermagem em unidade básica de saúde: percepção da equipe de enfermagem, demonstra que a equipe possui um entendimento razoável sobre a SAE e o PE, porém, ainda há dúvidas e conflitos de entendimento sobre os conceitos (Ribeiro, Padoveze, 2018). Acredita-se que a dificuldade em relação aos conceitos pode ser influenciada pela falta de consenso na própria literatura, pois, algumas vezes, os termos SAE e PE são tratados como sinônimos, em outras, não (Coren SP, 2015).

Os resultados evidenciaram que a SAE e o PE, não fazem parte da rotina diária da equipe e que não há um processo institucional estabelecido que permita a prática de maneira homogênea. O uso de tais metodologias é, portanto, ainda incipiente. Os achados indicam que há necessidade de um maior engajamento institucional, por meio do desenvolvimento de programas de capacitação e protocolos específicos, visando à minimização de barreiras e à potencialização de facilitadores do processo (Ribeiro, Padoveze, 2018).

De acordo com o estudo, a falta de uma linguagem universal é o que torna mais árduo o processo de enfermagem. As autoras relatam também que a visão do profissional acerca da SAE inserida na atenção primária é limitada, pois acreditam que a SAE é restrita à atenção terciária (Souza, 2015).

O artigo IV, O conhecimento do enfermeiro sobre a Sistematização da Assistência de Enfermagem: da teoria à prática, informa sobre a importância dos formulários, porque padronizam os registros e respalda legalmente as ações de enfermagem. As anotações devem conter termos técnicos, numa sequência lógica e objetiva, para que permita a continuidade do planejamento dos cuidados prestados. Mantê-los arquivados junto ao prontuário do cliente é útil para consulta de pesquisa e ensino, fonte de dados e para processos administrativos (Coren SP, 2000). Traz ainda, o grande problema da inexistência de formulários da SAE em metade das unidades de internação estudadas. Constatamos que mesmo onde eles existiam, alguns enfermeiros negaram o manuseio dos mesmos, indicando uma assistência distanciada da fundamentação teórica (Silva, 2011).

A sistematização das ações traria a autonomia ao enfermeiro, porém esta ideia, quando confrontada com a realidade do sistema de saúde atual, torna-se discordante, por causa de questões como mercado de trabalho, relação com outros profissionais de saúde, estrutura e organização política da saúde e educação, bem como as relações sociais e econômicas envolvidas no processo, que também interferem na almejada autonomia (Silva, Gomes, Anselmi, 1996).

O artigo X, Sistematização da assistência de enfermagem: subsídios para implantação, aborda as fases do planejamento para a implantação da SAE, sendo que elas revelam um processo bastante complexo, e que antes de mais nada, faz-se necessário conhecer a estrutura institucional onde ela será implantada (Hermida, Araújo, 2006).

Além disso, é preciso conhecer os aspectos que possam contribuir na sua implantação e os que podem prejudicá-la. Uma estratégia de marketing para "vender" a proposta da SAE pode estar centrada na melhoria da qualidade da assistência. Isso pode convencer chefias de enfermagem e a própria diretoria das instituições a "comprar" a ideia, especialmente se a instituição estiver em busca da qualidade nos serviços prestados aos pacientes (Hermida, Araújo, 2006). 
$\mathrm{O}$ artigo XI, Sistematização da assistência de enfermagem ao paciente crítico: proposta de instrumento de coleta de dados, nesse estudo evidenciamos apenas uma etapa da SAE, a coleta de dados, mas conhecendo a importância das demais (Bittar, Pereira, Lemos, 2006).

A coleta de dados tem por finalidade identificar os problemas reais ou potenciais do cliente, de forma a subsidiar o plano de cuidados e atender as necessidades encontradas prevenindo as complicações. É uma das etapas da SAE que mais exige tempo e trabalho, reunindo informações indispensáveis à comprovação da hipótese. É uma fase que pressupõe a confecção de um instrumento adequado de registro e leitura dos dados, e o desenvolvimento de técnicas para obter esses dados (Chizzotti, 1991).

Por fim, a coleta de dados é a base para o processo de enfermagem. Tem como objetivo identificar e obter importantes dados sobre o estado de saúde do indivíduo, família e comunidade. Essas informações são fundamentais para a individualização do plano de cuidados (Bittar, Pereira, Lemos, 2006).

O artigo XII, Autonomia e vulnerabilidade do enfermeiro na prática da Sistematização da Assistência de Enfermagem, com uma abordagem mais filosófica, consideram que a SAE é um caminho de autonomia para a profissão por representar uma metodologia de assistência reconhecida pelos enfermeiros, por permitir uma aproximação do enfermeiro junto ao paciente, tanto no momento da sua elaboração quanto na prestação do cuidado, sua maior competência; por exigir conhecimento científico, responsabilidade profissional e compromisso com o exercício profissional (Menezes, Priel, Pereira, 2011).

A questão do papel social do enfermeiro está identificada na Sistematização da Assistência de Enfermagem que se faz necessária para a avaliação crítica da pertinência e relevância do trabalho de enfermagem frente ao atendimento das necessidades de saúde (Barros, Chiesa, 2007).

A autonomia do enfermeiro ao praticar a SAE está no seu papel social, no cuidado que realiza ao paciente, nas orientações que faz para a equipe de enfermagem ao realizar a assistência, no atendimento à integralidade e individualidade do ser humano e nos resultados verificados pela instituição sobre o seu trabalho (Menezes, Priel, Pereira, 2011).

No artigo XIII, Sistematização da Assistência de Enfermagem em Unidade de Terapia Intensiva sustentada pela Teoria de Wanda Horta, percebe-se a importância da aplicação dos processos de enfermagem nas UTIs, pois só assim a Enfermagem é capaz de realizar uma assistência rápida e de qualidade. Na obra, os funcionários da UTI em questão pouco sabem sobre a SAE, e reconhecem ser necessário aprender mais, para poder aplicá-la na prática. Notou-se um grande interesse por parte de toda a equipe de enfermagem em conhecer melhor a SAE e torná-la parte de sua rotina (Amante, 2009).

Esse estudo identificou que os enfermeiros não sabiam utilizar os instrumentos pelo fato de não terem um contato prévio com os mesmos, além disso, os enfermeiros despendiam mais tempo realizando a SAE. A autora demonstra que não cabe apenas ao enfermeiro identificar o diagnóstico, mas também relacioná-lo com as características definidoras e com fatores relacionados (Amante, 2009).

De acordo com Pissaia, Da Costa, Moreschi, Rempel, Carreno e Granada (2018) a função da SAE é compreendida como um planejamento estratégico da assistência de enfermagem, que se embasa sob a perspectiva de uma teoria de enfermagem, sendo a das Necessidades Humanas Básicas preconizada no Brasil

Nacionalmente, a teoria das necessidades humanas básicas desenvolvida por Wanda de Aguiar Horta é um dos modelos mais estudados e conhecido pelos docentes de enfermagem devido a sua utilização como suporte teórico (Figueiredo, 2005).

A associação entre a teoria das Necessidades Humanas Básicas de Wanda Horta e Nanda abriu o horizonte dos participantes, pois a mesma além de organizar o serviço despertou o desejo de estudar e a sensação de se sentir parte da assistência, responsáveis pela assistência de enfermagem eficiente e individualizada (Amante, 2009).

$\mathrm{O}$ artigo XIV, Sistematização da assistência de enfermagem: facilidades e desafios do enfermeiro na gerência da assistência, abrange as instituições hospitalares e suas características específicas no que diz respeito às facilidades e desafios 
para a operacionalização da SAE, as quais devem ser analisadas pelos enfermeiros, a fim de que este instrumento assistencial seja implantado com conhecimento da situação real e com metas possíveis de serem alcançadas. O fluxo dos depoimentos apreendidos peregrinou para vislumbrar os cenários do cotidiano do enfermeiro em que ainda ocorre uma fragmentação no seu processo de trabalho, onde este profissional por tantos motivos não concretiza a SAE de forma sistematizada e individualizada (Soares, et al, 2015).

Assim, é notório enfatizar que na realidade das instituições hospitalares pesquisadas, por meio dos grupos focais, foram pontuadas várias situações em que, às vezes, o profissional deixa de exercer suas ações, por conta do que lhe é imposto diante das mudanças e transformações globais. Nesse sentido, fazendo uma articulação dos resultados, pode-se notar que existem mais desafios do que facilidades que perpassam no cotidiano do enfermeiro frente à operacionalização da SAE, tais como: implementar a SAE de maneira correta, a falta de impressos, protocolos, escassez de enfermeiros, o que gera a falta de tempo, a ausência de conhecimento, ou seja, a não capacitação dos profissionais, a falta de um ambiente para a passagem dos plantões, bem como os registros de enfermagem incompletos (Soares, et al, 2015).

No artigo XV, Desvelando a implementação da Sistematização da Assistência de Enfermagem, é reconhecido que a temática da Sistematização da Assistência de Enfermagem é atual embora seja discutida no Brasil desde a década de 70, com tentativas de implementação em diversas especialidades e contextos (hospitalar, ambulatorial, creche, etc). No entanto, percebese que essas tentativas nem sempre atingem o êxito desejado, pois "esbarram" em dificuldades das mais variadas origens, tornando a implementação da SAE um processo desestimulador e muitas vezes inviável na prática dos profissionais de enfermagem. Outras vezes ela persiste basicamente como uma atividade burocrática, perdendo toda a sua essência (Hermida, 2004).

Para uma efetiva implantação do PE, existem autores que consideram necessário haver primeiro um comprometimento da chefia de enfermagem com a proposta, promovendo reuniões e elaborando um plano de ação que incluiria: a sensibilização da equipe para a importância dessa metodologia; o desenvolvimento de um estudo aprofundado do tema com o envolvimento de toda a equipe; e a construção coletiva dos meios para viabilizar a execução do processo. Esses autores esclarecem que, o plano de ação viria garantir e assegurar que os objetivos fossem direcionados para viabilizar a implementação do PE, tendo como primeiro passo a sensibilização dos enfermeiros (Matté, Thofehrn, Muniz, 2001).

$\mathrm{O}$ artigo XVI, Sistematização da assistência de enfermagem na perspectiva dos enfermeiros: uma abordagem metodológica na teoria fundamentada, foi realizado em uma maternidade pública localizada na cidade de João Pessoa/PB. A escolha do local se justifica, em virtude de ser uma instituição reconhecida como centro de referência na formação e organização de profissionais de enfermagem no Estado da Paraíba, na área de obstetrícia e por desenvolver o processo de enfermagem em seu serviço (Medeiros, Santos, Cabral, 2012).

No estudo, os enfermeiros vêem a SAE como uma proposta para melhorar a qualidade da assistência prestada à clientela, através de um método que organiza, sistematiza e direciona todas as etapas do processo de cuidar, específico da enfermagem. Eles percebem como uma forma de aprofundar os conhecimentos, tanto formais quanto informais, conferindo autonomia na prática do enfermeiro, determinando sua importância dentro da profissão ou na ação conjunta com outros profissionais, trazendo mais visibilidade à profissão (Menezes, Priel, Pereira, 2011).

Por fim, a análise dos artigos revelou que existe uma quantidade insuficiente de profissionais da saúde nas unidades em geral, o que inviabiliza a adequação do sistema de trabalho aos modelos propostos para implementação da SAE. Desta forma, ao implementar a SAE a unidade precisa estabelecer padrões de qualidade no serviço prestado, no intuito de adequar a assistência sistematizada eficiente ao padrão que a instituição exige, condizente com o número de recursos humanos e materiais disponíveis (Casafus, 2013). 
Há necessidade de investimento em educação permanente e continuada para os profissionais da Atenção Básica, para que os mesmos possam se qualificar e aprimorar seus conhecimentos, a fim de se oferecer uma melhor assistência, pois a falta de conhecimento, de educação permanente e continuada e nomenclaturas utilizadas dificultam a implantação e implementação do SAE (Krauzer, 2015)

São necessários esforços e debates no meio assistencial, gestão, ensino e controle social, como propõe a Política Nacional de Educação Permanente, para o desenvolvimento de aspectos primordiais na qualificação da assistência em saúde, especialmente no âmbito da atenção básica, como a SAE (Krauzer, 2015).

\section{Conclusão}

A sistematização da assistência de enfermagem tornou-se a base da prática contemporânea na prestação de cuidados de enfermagem em diversas partes do mundo e um componente central do ensino da enfermagem. É reconhecida internacionalmente como um método que organiza e direciona o trabalho profissional, tendo como objetivo principal sistematizar e qualificar o atendimento ao paciente, família e comunidade. Por meio da SAE, o enfermeiro aplica seus conhecimentos técnicos e científicos para organizar, planejar, executar ações e instrumentalizar a equipe responsável pela assistência de enfermagem (Giehl, Costa, Pissaia, Moreschi, 2016).

Este estudo evidenciou artigos publicados entre 2004 e 2021, sendo que em todos foram apontadas dificuldades e possíveis soluções para a aplicação da SAE nas Unidades de Saúde.

Pode-se notar que existem mais desafios do que facilidades que perpassam no cotidiano do enfermeiro frente à operacionalização da SAE, tais como: implementar a SAE de maneira correta, a falta de impressos, protocolos, escassez de enfermeiros, o que gera a falta de tempo, a ausência de conhecimento, ou seja, a não capacitação dos profissionais, a falta de um ambiente para a passagem dos plantões, bem como os registros de enfermagem incompletos. Contudo, todos os participantes reconhecem a importância da SAE para uma assistência individualizada e qualificada, porém, esses entraves citados deixam o enfermeiro de mãos atadas, não tendo o respaldo necessário para implementá-la (Soares et al, 2015).

Apesar dos obstáculos para implementar a SAE, suas contribuições significativas devem ser levadas em conta, propondo um momento de reflexão pelos profissionais de enfermagem, especialmente os(as) enfermeiros(as). A Sistematização da Assistência de Enfermagem é compromisso e humanização do cuidado, sendo de grande valia o esforço para implementá-la nas unidades básicas, ILPS e unidades hospitalares.

Cabe destacar que nada adianta utilizar a SAE como receita de bolo, mas sim adequá-la de acordo com a realidade de cada instituição, sendo preciso verificar o número do pessoal de enfermagem proporcional ao número de leitos do hospital, contemplando à resistência por parte de alguns enfermeiros que recusam a SAE como respaldo legal da profissão, quebrando o tabu de que esse instrumento veio para somar e avigorar a autonomia do profissional (Soares et al, 2015).

Acredita-se que mais estudos sobre a temática sejam pertinentes para se ampliar o conhecimento na área, assim como, os resultados possam ser úteis para favorecer a discussão da temática e frisar a relevância da aplicação da SAE. (Barreto, 2020).

Conclui-se com este trabalho que a SAE é uma tarefa de suma importância que deveria ser realizada em todos os setores hospitalares, auxiliando no tratamento e cuidados referentes a cada paciente (Corbellini, Costa, Pissaia, 2019). Espera-se que a abordagem do tema neste estudo contribua preparando muitos enfermeiros para iniciarem o processo de implantação da SAE (Hermida, Araújo, 2006).

\section{Referências}

Amante, L. N, Rosseto, A. P., \& Schneider, D. G. (2009). Sistematização da Assistência de Enfermagem em Unidade de Terapia Intensiva sustentada pela Teoria de Wanda Horta. Rev. esc. enferm. USP, São Paulo, 43(1), 54-64, 
Barreto, M. S. et al (2020). Sistematização da assistência de enfermagem: a práxis do enfermeiro de hospital de pequeno porte. Rev Esc. Anna Nery, 24(4).

Barros, D. G., \& Chiesa, A. M. (2007). Autonomia e necessidades de saúde na Sistematização da Assistência de Enfermagem no olhar da saúde pública. Rev Esc Enferm USP.,41,:793-98.

Bittar, D. B, Pereira, L. V \& Lemos, R. A (2006). Sistematização da assistência de enfermagem ao paciente crítico: proposta de instrumento de coleta de dados. Rev Texto contexto - enferm., 15(4), 617-628.

Casafus, K. C. U et al (2013). Entre o êxito e a frustração com a Sistematização da Assistência de Enfermagem. Esc Anna Nery, 2 (17), 313- 321.

Carvalho, E. C et al (2009). Processo de enfermagem: resultados e consequências da utilização para a prática de enfermagem. Acta Paul Enferm. $22,554-7$.

Chaves, R. R., Silva, C. F, Motta, E. Ribeiro, E. D., \& Andrade, Y. N (2016). Sistematização da Assistência de Enfermagem: visão geral dos enfermeiros. Rev.Enferm UFPE, 10(4),1280-5.

Chizzotti, A. (1991). Pesquisa em ciências humanas e sociais. Cortez.

Cofen (2009). Resolução COFEN no 358/2009. Conselho Federal de Enfermagem.

Corbellini, B., Costa, A., \& Pissaia, L. (2019). Sistematização da assistência de enfermagem em pacientes com câncer de mama: a atuação do enfermeiro. Res., Soc, $8(9)$.

Coren-sp (2000). Decisão COREN-SP DIR/001/2000. Normatiza no Estado de São Paulo os princípios gerais para ações que constituem a Documentação de Enfermagem. São Paulo.

Coren- sp (2015). Principais legislações para o exercício da Enfermagem. Conselho Regional de Enfermagem de São Paulo.

Dal Sasso, G. T. M., et al (2013). Computerized nursing process: methodology to establish associations between clinical assessment, diagnosis, interventions, and outcomes. Rev Esc Enferm USP. 47(1):238-45.

Domingos, C. S., et al (2017). A aplicação do processo de enfermagem informatizado: revisão integrativa. Enfer Global 16(4):620-36.

Figueiredo, R. M et al (2005). Caracterização da produção do conhecimento sobre sistematização da assistência de enfermagem no Brasil. Rev Esc Enferm USP, 40(2): 299-303

Garcia, T. R (2016). Sistematização da assistência de enfermagem: aspecto substantivo da prática profissional. Esc. Anna Nery, 20(1), 5-10.

Gengo, et al (2018). Linkages of nursing diagnoses, outcomes, and interventions performed by nurses caring for medical and surgical patients using a decision support system. Int J Nurs Knowl, 29(4):269-75.

Giel, C., Costa, A., Pissaia, L., \& Moreschi, C. (2016). A equipe de Enfermagem frente ao processo de implantação da Sistematização da Assistência de Enfermagem. Rev.Enferm atenção à Saúde. 5(2), 87-95.

Gutiérrez, M. G., \& Morais, S. C. (2017). Sistematização da assistência de Enfermagem e a formação da identidade profissional. Rev. Bras Enferm. 70(2), 43641.

Hermida, P. M. \& Araújo, I. E. (2006). Sistematização da assistência de Enfermagem: subsídios para implantação. Rev.Bras Enferm. 59(5), 675-9.

Hermida, P. M (2004). Desvelando a implementação da Sistematização da Assistência de Enfermagem. Rev. bras. enferm., Brasília, $57(6), 733-737$.

Hohlfeld, T., Ritzhaupt, A. D., \& Barron, A. E. (2013). Are gender differences in perceived and demonstrated technology literacy significant? it depends on the model. Educ Technol Res Dev. 61(4):639-63.

Kang, Y., Hwang, W. J., \& Choi, J. (2019). A concept analysis of traditional Korean (Hanbang) nursing. Int J Nurs Knowl., 30(1):4-11.

Krauzer, I. M, Adamy, E..K, Ascari, R. A, Ferraz, L, Trindade, L. L \& Neiss, M (2015). Sistematização de enfermagem na atenção básica: o que dizem os enfermeiros? Ciencia Y Enfermeria, 21, (2): 31- 38.

Jesus, I. S, Sena, E, Meira, E. C, Gonçalves, L. H \& Alvarez, A. M (2010). Cuidado sistematizado a idosos com afecção demencial residentes em instituição de longa permanência. Rev. Gaúcha Enferm., 31(2), 285-92.

Matté, V. M, Thofehrn, M. B., \& Muniz, R. M. (2001). Opinião dos enfermeiros quanto à aplicabilidade do processo de enfermagem em unidade de tratamento intensivo. Revista Gaúcha de Enfermagem, 22(1):101-21.

Matiello, I., Costa, A., Lohmann, P., \& Lavall, E. (2020). Cuidados paliativos relacionados às doenças crônicas na terceira idade: uma revisão integrativa da literatura. Research, Society and Development, 9(7), e980974929.

Medeiros, A., Santos, S., \& Cabral, R. (2012). Sistematização da Assistência de Enfermagem na perspectiva dos enfermeiros: uma abordagem metodológica na teoria fundamentada. Rev. Gaúcha Enferm, 33(3), 174-181.

Menezes, S., Priel, M., \& Pereira, L. (2011). Autonomia e vulnerabilidade do enfermeiro na prática da Sistematização da Assistência de Enfermagem. Rev. esc. enferm. USP, 45(4), 953-958. 
Research, Society and Development, v. 10, n. 4, e8610413814, 2021

(CC BY 4.0) | ISSN 2525-3409 | DOI: http://dx.doi.org/10.33448/rsd-v10i4.13814

Morais, G., Brasil, T., Tolfo, G., Costa, A, \& Lohmann, P. (2020). A Sistematização da Assistência de Enfermagem (SAE) a um paciente com obesidade e hipertensão: relato de experiência. Research, Society and Development, 9(9), e962997940.

Oliveira, M. et al (2019). Sistematização da assistência de enfermagem: percepção e conhecimento da enfermagem Brasileira. Rev. Bras Enferm. Brasília, 72(6), 1547-1553.

Pereira, P, Dos Santos, F \& Rodrigues, M et al (2016). A família no cuidado ao idoso após o acidente por quedas. Rev Fund Care Online. 8(3):4717-4724.

Ribeiro, G \& Padoveze, M (2018). Sistematização da Assistência de Enfermagem em unidade básica de saúde: percepção da equipe de enfermagem. Rev. esc. enferm. USP, 52, e03375.

Salvador, P et al (2018). Validação de objeto virtual de aprendizagem para apoio ao ensino da sistematização da assistência de enfermagem. Rev. Bras. Enferm., 71(1), 11-19.

Santana, E et al (2021). Diagnósticos de enfermagem da taxonomia NANDA-I para idosos em instituição de longa permanência. Esc. Anna Nery, Rio de Janeiro, 25(1), e20200104.

Silva, E et al (2011). O conhecimento do enfermeiro sobre a Sistematização da Assistência de Enfermagem: da teoria à prática. Rev. esc. enferm. USP, 45(6), 1380-1386.

Silva, E., Gomes, E., \& Anselmi, M. (1993). Enfermagem: realidade e perspectiva na assistência e no gerenciamento. Rev Lati no Am Enferm, 1(1):59-63.

Silva, R et al (2018). Linkagesofnursing diagnoses, outcomes, andinterventionsperformedby nurses caring for medical andsurgicalpatientsusing a decisionsupport system. Int J NursKnowl., 29(4), 269-75.

Silva, T, Santos, R, Crispim, L \& Almeida, L (2016). Conteúdo dos registros de enfermagem em hospitais: contribuições para o desenvolvimento do processo de enfermagem. Rev. Enfermagem em Foco. 7(1).

Soares, M et al (2015). Sistematização da assistência de enfermagem: facilidades e desafios do enfermeiro na gerência da assistência. Esc. Anna Nery, 19(1), 47-53.

Somariva, V., Birolo, I., Tomasi, C., \& Soratto, J. (2019). Percepções das equipes de enfermagem na Atenção Básica frente a Sistematização da Assistência de Enfermagem. Rev Enferm Foco, 10 (4): 142-147.

Souza, L., Santana, I., \& Jesus, S. (2017). Capacidade funcional de idosos residentes em uma instituição de longa permanência. Acta Biomed Bras.,8(2):10110.

Souza, N., Costa, B., Carneiro, D., Barbosa, H., \& Santos, I. (2015). Sistematização da Assistência de Enfermagem: dificuldades referidas por enfermeiros de um hospital universitário. Revista de Enfermagem- UFPR, 3(9). 7104-10. 